\title{
General Psychiatry Face and object visual working memory deficits in first-episode schizophrenia correlate with multiple neurocognitive performances
}

\author{
Yi Liu, ${ }^{1}$ Taiyong Bi, ${ }^{2}$ Bei Zhang, ${ }^{1,3}$ Qijie Kuang, ${ }^{1}$ Haijing Li, ${ }^{1}$ Kunlun Zong, ${ }^{1}$ \\ Jingping Zhao, ${ }^{4}$ Yuping Ning, ${ }^{1}$ Shenglin She, ${ }^{1}$ Yingjun Zheng ${ }^{1}$
}

To cite: Liu Y, Bi T, Zhang B, et al. Face and object visual working memory deficits in first-episode schizophrenia correlate with multiple neurocognitive performances. General Psychiatry 2021;34:e100338. doi:10.1136/ gpsych-2020-100338

Received 16 July 2020 Revised 19 November 2020 Accepted 15 December 2020

Check for updates

(c) Author(s) (or their employer(s)) 2021. Re-use permitted under CC BY-NC. No commercial re-use. See rights and permissions. Published by BMJ.

${ }^{1}$ Department of Psychiatry, The Affiliated Brain Hospital of Guangzhou Medical University, Guangzhou, Guangdong, China ${ }^{2}$ Centre for Mental Health Research in School of Management, Zunyi Medical University, Zunyi, Guizhou, China ${ }^{3}$ Department of Psychology, General and Experimental Psychology, LMU Munich, Germany

${ }^{4}$ Mental Health Institute of the Second Xiangya Hospital, Central South University: Chinese National Clinical Research Center on Mental Disorders; Chinese National Technology Institute on Mental Disorders; Hunan Key Laboratory of Psychiatry and Mental Health, Changsha, Hunan, China

Correspondence to Dr Yingjun Zheng; brainzheng@gzhmu.edu.cn

\section{ABSTRACT}

Background Working memory (WM) deficit is considered a core feature and cognitive biomarker in patients with schizophrenia. Several studies have reported prominent object WM deficits in patients with schizophrenia, suggesting that visual WM in these patients extends to non-spatial domains. However, whether non-spatial WM is similarly affected remains unclear.

Aim This study primarily aimed to identify the processing of visual object WM in patients with first-episode schizophrenia.

Methods The study included 36 patients with firstepisode schizophrenia and 35 healthy controls. Visual object WM capacity, including face and house WM capacity, was assessed by means of delayed matchingto-sample visual WM tasks, in which participants must distribute memory so that they can discriminate a target sample. We specifically examined their anhedonia experience by the Temporal Experience of Pleasure Scale and the Snaith-Hamilton Pleasure Scale. Cognitive performance was measured by the Repeatable Battery for the Assessment of Neuropsychological Status (RBANS).

Results Both face and house WM capacity was significantly impaired in patients with schizophrenia. For both tasks, the performance of all the subjects was worse under the high-load condition than under the low-load condition. We found that WM capacity was highly positively correlated with the performance on RBANS total scores $(r=-0.528, p=0.005)$, RBANS delayed memory scores $(r=-0.470, p=0.013)$, RBANS attention scores $(r=-0.584$, $p=0.001)$, RBANS language scores $(r=-0.448, p=0.019)$, Trail-Making Test: Part A raw scores $(r=0.465, p=0.015)$ and simple IQ total scores $(r=-0.538, p=0.005)$, and correlated with scores of the vocabulary test $(r=-0.490$, $p=0.011)$ and scores of the Block Diagram Test $(r=-0.426$, $\mathrm{p}=0.027$ ) in schizophrenia. No significant correlations were observed between WM capacity and Positive and Negative Syndrome Scale symptoms.

Conclusions Our research found that visual object WM capacity is dramatically impaired in patients with schizophrenia and is strongly correlated with other measures of cognition, suggesting a mechanism that is critical in explaining a portion of the broad cognitive deficits observed in schizophrenia.

\section{INTRODUCTION}

Schizophrenia is a severe, chronic and socially disabling mental disorder. Patients with schizophrenia suffer from delusions, hallucinations and multiple cognitive deficits. Working memory (WM) deficit is considered a core feature and cognitive biomarker in patients with schizophrenia. ${ }^{1-6}$

WM, a cognitive system that is responsible for temporarily holding information available for processing, is important for reasoning, decision-making and social behaviour. ${ }^{7} \mathrm{WM}$ has been considered the central foundation of human high-level cognitive activity. It enables the interactions of multiple information from memory, sensory experience and motor control into process-oriented behaviour. ${ }^{8}$ The disturbance of this system can result in dysfunction of cognitive organisation, failure of self-monitoring, distractibility and other phenotypical features of schizophrenia.

WM involves three phases: novel information encoding; maintenance, updating and manipulation; and information retrieval. ${ }^{9-12}$ Researchers have demonstrated experimentally that neurons in the prefrontal cortex generate persistent firing to retain information. ${ }^{13} 14$ A number of studies have documented WM deficits in patients with schizophrenia. Zhang et al examined factors that exhibited influence on visual WM and demonstrated relationships between visual WM and multiple hospitalisations and the suffering caused by severe negative symptoms in patients with adult-onset schizophrenia. ${ }^{15}$ Gan et al found that patients with schizophrenia showed poor performance in the n-back task than patients with methamphetamine-associated psychosis. $^{16}$ Studies on specific aspects of WM may provide pivotal evidence for the aetiology of a disease. 
Functional MRI (fMRI) studies also provided detailed evidence that the superior part of the ventrolateral prefrontal cortex (VLPFC) is likely involved in encoding of visual WM information ${ }^{17}{ }^{18}$; the dorsolateral prefrontal cortex (DLPFC) is crucial for maintenance ${ }^{19-21}$ and the inferior portion of the VLPFC is particularly important for retrieval. ${ }^{22}{ }^{23}$ fMRI studies have suggested that visuospatial WM impairment is associated with reduced prefrontal neuronal activation. ${ }^{24-26}$ Schlösser et al reported a reduced activation in the anterior cingulate cortex and the DLPFC in patients with schizophrenia during the encoding phase of WM. ${ }^{27}$ The meta-analysis of fMRI studies has consistently documented an abnormal activation in the DLPFC during WM in patients with schizophrenia. ${ }^{27-29}$ Additionally, positron emission tomography has revealed a functional disconnection in prefrontal-parietal networks during WM processing in these patients. ${ }^{30}$

Their findings have confirmed that WM is a robust feature of schizophrenia across different paradigms, especially in visuospatial WM tasks. ${ }^{25}{ }^{26}$ However, whether the visual WM deficit extends to non-spatial domains remains unknown. Several studies have reported prominent object WM deficits in patients with schizophrenia, suggesting that visual WM in these patients extends to non-spatial domains. ${ }^{31-33}$

This study primarily aimed to identify the processing of visual object WM, including house and face WM, processing under different condition levels of memory load in patients with first-episode schizophrenia. Studies of non-spatial WM could greatly advance our understanding of the capacity of visual WM in patients with schizophrenia. Further, the two visual tasks we applied could be used to distinguish different types of visual stimuli, especially in recognition of face specificity. ${ }^{32} 34$ Additionally, this study was designed to explore the relation patterns between WM capacity, including clinical symptoms and other functions, during visual object WM processing in patients with schizophrenia in comparison with healthy control participants. We hypothesised that patients with schizophrenia would show a reduced visual object WM processing and association with cognitive performances.

\section{METHODS}

\section{Subjects}

Of the final 71 subjects recruited and divided into two groups, 36 were patients with first-episode schizophrenia and 35 were healthy controls. All the subjects were collected from the Affiliated Brain Hospital. Patients with schizophrenia were diagnosed by experienced clinical psychiatrists. Diagnostic and Statistical Manual of Mental Disorders, Fourth Edition (DSM-IV) ${ }^{35}$ criteria were used to confirm the diagnosis of patients with schizophrenia.

All the participants were subjected to stringent inclusion pipeline management in accordance with the following criteria: (1) subjects should be within the age range of 17-50 years in both groups; (2) subjects in the patients group were all diagnosed with schizophrenia for the first time, with illness duration of no more than 2 years, and they received antipsychotic therapy in less than 1 year before the time of enrolment; (3) subjects in the control group were collected from the community, did not meet the criteria set by the Criteria of Psychosis-Risk Syndromes or DSM-IV and had no documented family history of psychiatric problems or medications; and (4) subjects received formal education of no less than 6 years; (5) subjects had normal or corrected-to-normal vision and (6) subjects were right-handed.

The exclusion criteria for the subjects were as follows: (1) subjects who met DSM-IV criteria for any psychotic disorder or had delirium, dementia, amnesia or other severe cognitive impairment in the past or intellectual developmental disabilities $(\mathrm{IQ}<70)$ before their schizophrenia diagnosis was made; (2) clinically significant somatic diseases; (3) substance abuse in the past 3 months; (4) subjects with a documented history of brain injury, epilepsy or other known diseases of the central nervous system; (5) subjects who underwent MECT (Modified Electric Convulsive Therapy) in the past 6 months; (6) women subjects with a documented history of recent pregnancy or abortion and (7) subjects with hearing impairments.

\section{Cognitive and general psychiatric assessments}

All the participants received a demographic questionnaire about age, gender, degree of education, marriage and family history of mental illness. The psychotic symptoms of the patients with schizophrenia were assessed by two trained psychiatrists or psychologists using the Positive and Negative Syndrome Scale (PANSS). ${ }^{36}$ The Temporal Experience of Pleasure Scale ${ }^{37}$ and the Snaith-Hamilton Pleasure Scale ${ }^{38}$ were applied to test the anhedonia experience of patients with schizophrenia. We further examined the relationship between anhedonia experience and cognitive performance of schizophrenia. The Personal and Social Performance Scale ${ }^{39}$ was used to test the social functioning of these patients. The antipsychotic medication status, including drug types, duration of medication therapy and chlorpromazine equivalent doses at the time of testing, is recorded in table 1 . The auditory hallucination conditions of all the patients were also assessed (see flowchart in figure 1).

Simple IQ tests, including Block Diagram Test and Vocabulary Test, were administered to examine the innate and acquired IQ of all the subjects. Repeatable Battery for the Assessment of Neuropsychological Status (RBANS) ${ }^{40}{ }^{41}$ Trail-Making Test: Part A (TMT-A) and Mayer-Salovey-Caruso Emotional Intelligence Test (MSCEIT): Managing Emotions tests in the MATRICS Consensus Cognitive Battery ${ }^{42}{ }^{43}$ were used to evaluate the cognitive performance of each subject. Cognitive evaluation was carried out by trained and certified psychiatrists, psychologists or neuropsychological examiners. 
Table 1 Demographic and clinical characteristics

\begin{tabular}{|c|c|c|c|c|c|c|}
\hline \multirow[b]{2}{*}{ Characteristics } & \multicolumn{2}{|r|}{ SCZ } & \multicolumn{2}{|r|}{ CON } & \multicolumn{2}{|c|}{ Statistics } \\
\hline & $\mathbf{n}$ & Mean (SD) & $\mathbf{n}$ & Mean (SD) & $\mathbf{t}$ & $P$ value \\
\hline Age & 31 & $25.0(6.8)$ & 33 & $24.2(5.0)$ & $t=0.486$ & 0.629 \\
\hline Year of education & 31 & $11.1(3.3)$ & 33 & $11.7(3.1)$ & $\mathrm{t}=-0.716$ & 0.477 \\
\hline Married (Y/N) & 31 & $11 / 20$ & 33 & $8 / 25$ & & \\
\hline Simple IQ & 30 & $96.1(13.2)^{\star \star}$ & 33 & $106.2(12.1)$ & $t=3.155$ & 0.002 \\
\hline Block Diagram Test & 31 & $8.9(2.7)^{\star \star \star}$ & 33 & $11.5(2.5)$ & $\mathrm{t}=-3.879$ & $<0.001$ \\
\hline Vocabulary & 30 & $9.7(2.7)$ & 33 & $10.7(2.2)$ & $t=-1.650$ & 0.104 \\
\hline RBANS total & 31 & $368.3(61.7)^{\star \star \star}$ & 32 & $439.7(49.8)$ & $t=-5.061$ & $<0.001$ \\
\hline Attention & 31 & $92.7(17.9)^{\star}$ & 32 & $103.1(13.5)$ & $\mathrm{t}=-2.593$ & 0.012 \\
\hline Delayed memory & 31 & $70.1(17.9)^{\star \star \star}$ & 33 & $85.6(10.9)$ & $\mathrm{t}=-4.204$ & $<0.001$ \\
\hline TMT-A & 31 & $52.3(19.7)^{\star \star}$ & 33 & $38.6(13.4)$ & $\mathrm{t}=-3.282$ & 0.002 \\
\hline MSCEIT & 31 & $80.3(9.6)$ & 33 & $84.1(6.8)$ & $t=1.840$ & 0.071 \\
\hline TEPS total & 29 & $67.8(11.7)$ & 33 & $72.8(9.4)$ & $t=-1.891$ & 0.063 \\
\hline SHAPS total & 31 & $30.4(5.3)$ & 33 & $32.9(5.2)$ & $t=-1.885$ & 0.064 \\
\hline Duration of illness (months) & 31 & $8.5(7.0)$ & & & & \\
\hline Age of first episode & 31 & $24.0(6.8)$ & & & & \\
\hline
\end{tabular}

Medication status at time of testing

\begin{tabular}{lrl}
\hline None medicated & 8 \\
\hline Antipsychotic & 20 & \\
\hline Atypical & 18 & \\
\hline Atypical+traditional & 2 & \\
\hline Antidepression & 1 & \\
Unknown medication & 2 & \\
\hline Duration of medication treatment (days) & 31 & $26.4(37.2)$ \\
\hline Chlorpromazine & 31 & $222.1(244.4)$ \\
equivalents (mg) & 31 & $65.6(15.0)$ \\
\hline PANSS total & 31 & $17.6(5.1)$ \\
\hline PANSS positive & 31 & $13.7(4.1)$ \\
\hline PANSS negative & 31 & $34.3(8.6)$ \\
\hline PANSS general & 31 & $55.4(15.3)$ \\
\hline PSP total & 31 & $3 / 28$ \\
\hline Past hospitalisation history $(\mathrm{Y} / \mathrm{N})$ & 31 & $20 / 11$ \\
\hline Auditory hallucination $(\mathrm{Y} / \mathrm{N})$ & \\
\hline
\end{tabular}

Levels of significance: ${ }^{*} \mathrm{p}<0.05,{ }^{* *} \mathrm{p}<0.01,{ }^{* * *} \mathrm{p}<0.001$

CON, healthy controls; F, female; L, left; M, male; MSCEIT, Mayer-Salovey-Caruso Emotional Intelligence Test; N, no; PANSS, Positive and Negative Syndrome Scale; PSP, Personal and Social Performance Scale; R, right; RBANS, Repeatable Battery for the Assessment of Neuropsychological Status; SCZ, patients with schizophrenia; SHAPS, Snaith-Hamilton Pleasure Scale; TEPS, Temporal Experience of Pleasure Scale; TMT-A, TrailMaking Test: Part A; Y, yes.

\section{Stimuli and procedures of WM experiments}

Our research aimed to investigate the visual WM capacity of patients with schizophrenia and the healthy controls. The experiments were performed in a quiet, dimly lit room. The tasks were programmed in a custom-made
MATLAB (MathWorks) algorithm, and the stimuli were generated with the Psychtoolbox V.3.0 for MATLAB (http://psychtoolbox.org).

A delayed matching-to-sample (DMTS) paradigm was applied to administer visual WM experiments. Face and 


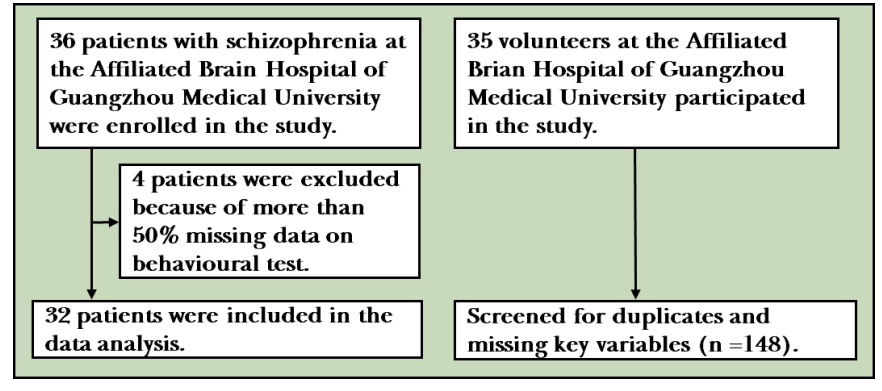

Figure 1 Flowchart.

house stimuli were included; high and low conditions of memory load were designed in each experiment. During each task, the visual WM stimuli were presented on a 19-inch Dell LCD monitor at a nominal viewing distance of $60 \mathrm{~cm}$ with a screen spatial display resolution of $1024 \times 768 \mathrm{DPI}$ and a $60 \mathrm{~Hz}$ refresh rate. All the subjects were instructed to sit comfortably throughout the whole experiment and to keep their eyes fixated on the small white fixation cross displayed on the centre of the monitor before the next trial and rest interval.

\section{House WM experiment}

In the house task, 64 house pictures were selected from the internet via Google and other search engines. Photoshop was used to match the root mean square contrast and brightness of these house pictures. All the pictures were of the same size, extended to $1.93^{\circ} \times 2.10^{\circ}$ and converted to grayscale.

The house WM experiment contained three blocks. Each block included 40 trials in which one-half was performed under the low-load condition, whereas the other half was performed under the high-load condition. One house sample was included under the condition of low visual memory load, whereas two house samples were included under the condition of high visual memory load. Trials began with the presentation of a fixation cross on the monitor screen for 1000-2000 ms random duration. Subsequently, one or two samples were presented on the monitor screen for $600 \mathrm{~ms}$. All the participants were required to try their best to maintain the content of the houses presented on the monitor for $3000 \mathrm{~ms}$ before

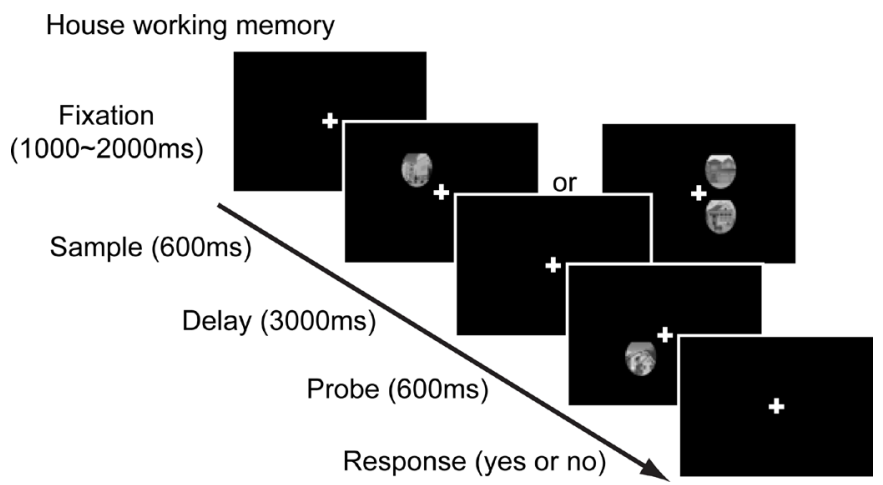

Figure 2 Example of working memory task under low-load (one sample picture) or high-load (two sample pictures) condition of house stimulus. recognition. Each sample was displayed at the four positions relative to the fixation on the centre of the screen: top-left, bottom-left, top-right and bottom-right. Additionally, the centre of each sample was set $2.47^{\circ}$ away from the central fixation cross during the encoding phase. The target probe house was then presented on the screen for $600 \mathrm{~ms}$. All the participants were asked to judge whether the target matched one of the original samples presented during the encoding phase. The subjects were instructed to press the ' $n$ ' key if the probe matched one of the samples as fast and accurately as possible and to press the ' $m$ ' key if the probe did not match one of the samples (see the procedure in figure 2). Each participant has free time to make response. Under each load condition, the probe and the samples were half matched. In each block, the order of the four kinds of houses in these trials was counterbalanced (low or high by matched or unmatched).

\section{Face WM experiment}

The procedure of the face WM task was the same as that of the house WM experiment, only the house pictures were replaced with face pictures. The 64 face samples applied were selected from the Chinese facial affective picture system. ${ }^{44}$ These faces included 32 women and 32 men with a neutral expression, and their outer features (ears, hairs and face contour) were excluded.

\section{Statistical analysis}

Statistical analyses were conducted in SPSS V.23. Differences in demographics (age, gender and education) and clinical and cognitive data were compared between groups by using independent-samples t-tests and $\chi^{2}$ tests. Mixed model analyses of variance (ANOVAs) were conducted to analyse the data on reaction time (RT) and accuracy from the visual WM experiments that involved two visual WM stimuli (house and face) under two memory load conditions (high and low) in the two groups (patients with schizophrenia and healthy controls). Pearson correlations were performed between the behavioural data and the performance, including clinical symptoms, medication dosages and cognitive function. A post hoc test was conducted for the correlations. Protected correction levels were Benjamini and Hochberg's ( $\mathrm{BH})$ corrected significance level (at $\alpha=0.05$ ) for correlations between a specific variable and the data on accuracy and RT.

\section{RESULTS}

\section{Demographic, clinical and cognitive characteristics}

A total of 67 subjects completed the behavioural experiments for analysis. This data set included 32 patients with schizophrenia and 35 healthy controls. Demographic and clinical data, which include age, gender, years of education, medication status at time of testing and PANSS symptom rating, are presented in table 1. Age, gender or education did not significantly differ between the subjects of the two groups. In this sample, the patients had the diagnosis of schizophrenia for an average of 8.5 months. 
Table 2 Behavioural results

\begin{tabular}{|c|c|c|c|c|c|}
\hline \multirow[b]{2}{*}{ Group } & \multirow{2}{*}{$\begin{array}{l}S C Z \text { ( } n=32 \text { bv) } \\
\text { Mean (SD) }\end{array}$} & \multirow{2}{*}{$\begin{array}{l}\text { CON (n=35) } \\
\text { Mean (SD) }\end{array}$} & \multicolumn{3}{|c|}{ Statistics } \\
\hline & & & $t$ & df & $P$ value \\
\hline Face low-load RT & $0.873(0.467)^{\star \star}$ & $0.596(0.268)$ & -2.940 & 65 & 0.004 \\
\hline Face low-load accuracy & $0.770(0.126)^{\star \star}$ & $0.875(0.114)$ & 3.561 & 65 & 0.001 \\
\hline Face high-load accuracy & $0.610(0.083)^{\star \star \star}$ & $0.702(0.073)$ & 4.873 & 65 & $<0.001$ \\
\hline House low-load accuracy & $0.772(0.152)^{\star \star \star}$ & $0.900(0.106)$ & 3.976 & 65 & $<0.001$ \\
\hline House high-load accuracy & $0.640(0.084)^{\star *}$ & $0.704(0.092)$ & 2.979 & 65 & 0.004 \\
\hline
\end{tabular}

Levels of significance: ${ }^{* *} p<0.01,{ }^{* * *} p<0.001$.

$\mathrm{CON}$, healthy controls; RT, reaction time; SCZ, patients with schizophrenia.

The treatment doses of antipsychotics were converted to their equivalent in chlorpromazine dosage.

\section{Behavioural data}

Accuracy and RT were calculated and used to evaluate the WM performance of the subjects. Four subjects in the schizophrenia group were excluded from the final statistical analyses because of more than 50\% missing data on behavioural tests. The accuracy and RT data were subjected to two conditions (face and house) by two memory load conditions (high and low) and by the two groups' (patients with schizophrenia and healthy controls) ANOVAs.

Behavioural results are presented in table 2. ANOVA on accuracy revealed the main significant effects of group $(\mathrm{F}=56.49, \mathrm{p}<0.001)$ and memory load $(\mathrm{F}=165.14$, $\mathrm{p}<0.001)$. The accuracy of the patients with schizophrenia was lower than that of the controls under a certain memory load condition. The stimuli had no main effect $(\mathrm{F}=1.29, \mathrm{p}=0.257)$. In addition, no significant interaction was observed between group and memory load $(\mathrm{F}=2.13$, $\mathrm{p}=0.146)$ or between group and stimulus task $(\mathrm{F}=0.008$, $\mathrm{p}=0.927$ ). ANOVA on $\mathrm{RT}$ also showed the main significant effects of group $(\mathrm{F}=43.80, \mathrm{p}<0.001)$ and memory load $(\mathrm{F}=7.55, \mathrm{p}=0.006)$. The RT of the patients with schizophrenia was slower than that of controls under a certain
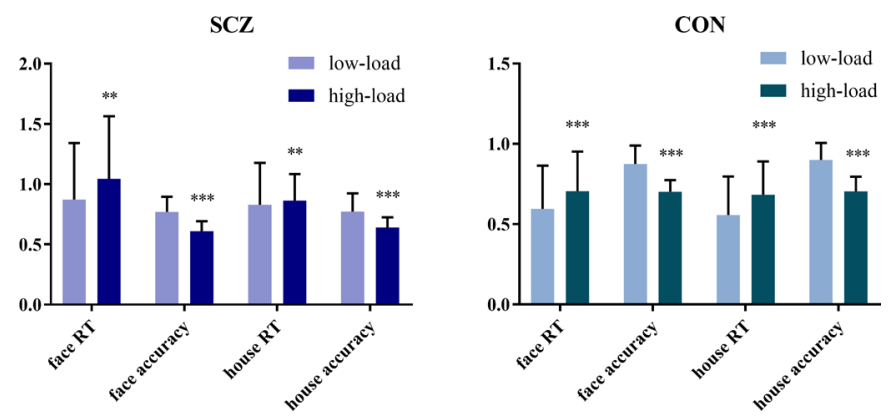

Figure 3 Face and house working memory across different conditions of memory load in group of patients with schizophrenia and controls. Levels of significance: ${ }^{* *} \mathrm{p}<0.01$, ${ }^{* * *} \mathrm{p}<0.001$. CON, healthy control; RT, reaction time; SCZ, patient with schizophrenia. memory load condition. The stimuli had no main effect $(\mathrm{F}=2.86, \mathrm{p}=0.092)$. In addition, no significant interaction was observed between group and memory load $(\mathrm{F}=0.037$, $\mathrm{p}=0.848)$ or between group and stimulus $(\mathrm{F}=0.966$, $\mathrm{p}=0.327)$.

The main significant effect of memory load indicated that memory load influenced the visual WM capacity of all the participants. The accuracy of the patients and the controls was lower under the high-load memory condition than under the low-load memory condition. The $\mathrm{RT}$ of the patients and the controls was slower under the high-load memory condition than under the low-load memory condition (see figure 3 ).

The stimuli showed no main effects on RT and accuracy. As such, further comparisons were made between the two stimuli in each group. However, the accuracy of the patients with schizophrenia $(\mathrm{t}=2.376, \mathrm{df}=31, \mathrm{p}=0.024)$ and the controls $(\mathrm{t}=3.104, \mathrm{df}=34, \mathrm{p}=0.004)$ was lower in the face WM task compared with that in the house WM task (see details in figure 4).

\section{Correlations}

Pearson correlation analysis was performed between the WM capacity and these features, including clinical symptoms, medication dosages, illness duration and cognitive performances, in patients with schizophrenia.

The results showed no significant correlations survived the Benjamini and Hochberg (BH)-protected correction between WM capacity and any clinical features in patients with schizophrenia. Correlations that survived the BH-protected correction were found between WM capacity and cognitive performances. The significant correlations between the WM capacity and cognitive performances were as follows: RT under the low-load condition of the face task was correlated with RBANS total scores $(\mathrm{r}=-0.528, \mathrm{p}=0.005)$, RBANS delayed memory scores $(\mathrm{r}=-0.470, \mathrm{p}=0.013)$, RBANS attention scores $(\mathrm{r}=-0.584$, $\mathrm{p}=0.001)$, RBANS language scores $(\mathrm{r}=-0.448, \mathrm{p}=0.019)$, TMT-A raw scores $(\mathrm{r}=0.465, \mathrm{p}=0.015)$ and simple IQ total scores $(\mathrm{r}=-0.538, \mathrm{p}=0.005)$ and correlated with scores of the vocabulary test $(\mathrm{r}=-0.490, \mathrm{p}=0.011)$ and scores of 


\section{Accuracy}

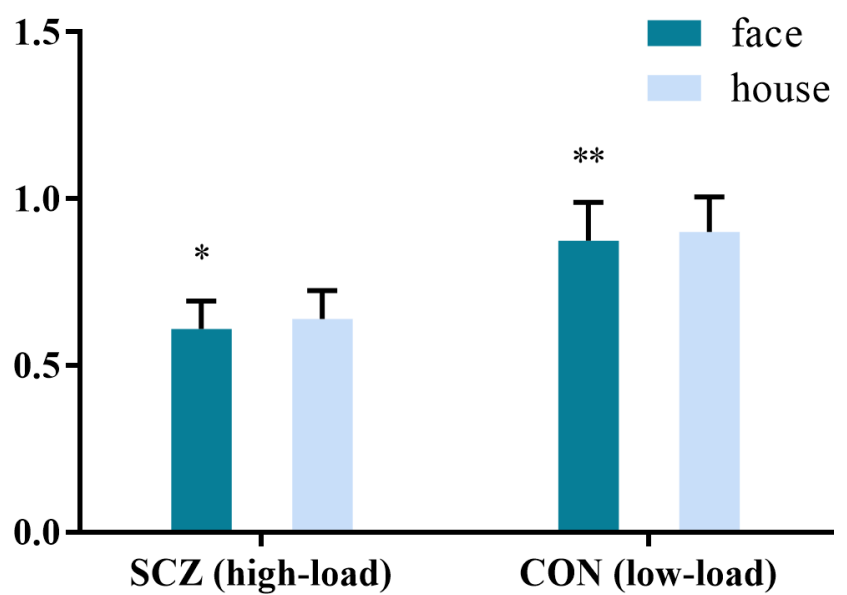

Figure 4 Accuracy of face and house working memory task in groups of patients with schizophrenia and healthy controls. ${ }^{*} \mathrm{P}<0.05,{ }^{* *} \mathrm{P}<0.01$. CON, healthy control; SCZ, patient with schizophrenia.

the Block Diagram Test $(\mathrm{r}=-0.426, \mathrm{p}=0.027)$. Our study observed that no significant correlation survived the $\mathrm{BH}-$ protected correction between WM capacity and clinical features.

\section{DISCUSSION}

\section{Main findings}

This study aimed to explore visual object WM capacity in patients with schizophrenia. The WM performance of all the subjects was worse under the high-load condition than under the low-load condition in the two tasks. The RT of all the participants showed no significant differences between the house or face task. However, the accuracy of all the participants was worse during the face task than during the house task. Additionally, face WM capacity was significantly correlated with multiple cognitive performances. No correlations were observed between WM capacity and clinical features. These results indicated that the house and face WM capacity of patients with schizophrenia were significantly impaired compared with that of controls, and a common mechanism explains impaired WM capacity and cognitive deficits in schizophrenia.

Both house and face WMs were significantly impaired in patients with schizophrenia. Additionally, the performances under the high-load condition were lower than that under the low-load condition. Given the poor performance under high-load condition in the control group as well, this result may be attributed to the difficulty of the high-load task. However, the lack of group by load interaction indicated that the group of patients with schizophrenia was not disproportionately influenced by this difference in load condition during the house and face WM tasks compared with that of the control group. Our observations were consistent with those in previous studies, which reported a variety of object WM deficits found in object WM tasks, such as DMTS tasks for familiar object and novel shapes ${ }^{31-33}$ and object WM tasks with increasing difficulty, ${ }^{45}$ suggesting that these two components are affected in schizophrenia.

Previous studies have shown that the temporal lobe region plays vital roles in object perception. Lesions in this brain region often result in abnormalities in recognition, identification and naming of different categories of objects. ${ }^{46}{ }^{47}$ Neuroimaging evidence supports the concept that neurons in different areas of the human temporal lobe have properties that are specialised for different categories of objects. For instance, the response of the fusiform face area (FFA) to images of faces is higher than its response to a variety of non-facial objects. ${ }^{4-52}$ By contrast, the response of the parahippocampal place area (PPA) to images of places, visually presented words, body parts and scenes is higher than its response to faces. ${ }^{53-55}$ Our study revealed significant house and face WM deficits in patients with schizophrenia possibly resulting from cortical deficits of the FFA and the PPA observed in schizophrenia. Furthermore, the performance of the patients with schizophrenia and the controls during the face task was worse than that during the house task. This finding might be in accordance with the concept that different areas of the temporal cortex are selective to facial and building recognition.

Additionally, we observed that the WM performance (RT) under the low-load condition of the face task was negatively correlated with multiple cognitive performances, including processing speed, attention, language and delayed memory deficits, in patients with schizophrenia. Consistent with our finding, previous studies also indicated deficits in multiple cognitive domains in patients with schizophrenia. ${ }^{56}{ }^{57}$ Additionally, Zhang et al also demonstrated that Chinese Han subjects with first episode of schizophrenia showed more severe neurocognitive deficits in the domain of speed of processing. ${ }^{58}$ These results suggested that longer RT correlated with more impaired cognitive functions. The $r^{2}$ of correlations of RT with intelligence, processing speed, attention, language and delayed memory ranged from 0.16 to 0.36 , which indicated that a portion of cognitive deficits might explain the poor performance during the face WM task. The WM performance under house task was not significantly correlated with cognitive performance, possibly indicating that cognitive deficits might contribute less to the dysfunction of house WM. Collectively, these observations might suggest that processing a stream of face and house WMs depended on distributed brain regions. Our study observed no significant correlation between face or house WM deficits and clinical symptoms and antipsychotic medication doses. This result is perhaps due to most of the patients recruited in our study being in the remission state with low scores of positive and negative symptoms. Previous studies have demonstrated correlations between object WM capacity and the negative symptoms of patients with schizophrenia in the partial remission stage. ${ }^{32}$ 


\section{Limitations}

This study was limited by the cross-sectional design of small samples. Future studies with large samples and follow-up studies should be designed to further explore the visual WM deficits in patients with first-episode schizophrenia and the relation with neurocognitive performances.

\section{Implications}

Visual WM capacity was examined using a well-validated visual WM task. Our findings indicated that patients with schizophrenia showed significant house and face WM deficits under low-load and high-load conditions of memory relative to healthy controls. Additionally, face and house WM capacities were not related to clinical symptoms, duration of illness or medication but showed high and broad correlations with impaired cognitive function. This research found that visual WM is dramatically impaired in patients with schizophrenia and is strongly correlated with other measures of cognition, suggesting a mechanism that is critical in explaining a portion of the broad cognitive deficits observed in schizophrenia. However, future studies should combine fMRI to elucidate the brain mechanism of changes in visual WM in patients with schizophrenia.

Acknowledgements The authors acknowledge the National Natural Science Foundation of China and the Medical Scientific Research Foundation of Guangzhou in Guangdong Province of China for financial support.

Contributors All authors contributed to and approved the final manuscript. YL, $T B, S S$ and $Y Z$ designed the study; YL wrote the protocol and the first draft of the manuscript; QK, HL and KZ collected the original imaging data; $\mathrm{YL}$ and SS managed and analysed the imaging data and ran the statistical analysis.

Funding This work was supported by grants from the National Natural Science Foundation of China (grant 81671334), Planed Science and Technology Projects of Guangzhou (grant 201904010267) and the Medical Scientific Research Foundation of Guangzhou in Guangdong Province of China (grant 2060404).

Competing interests None declared.

Patient consent for publication Not required.

Ethics approval This study was approved by the institutional review board of the Affiliated Brain Hospital of Guangzhou Medical University.

Provenance and peer review Not commissioned; externally peer reviewed.

Data availability statement Data are available upon reasonable request.

Open access This is an open access article distributed in accordance with the Creative Commons Attribution Non Commercial (CC BY-NC 4.0) license, which permits others to distribute, remix, adapt, build upon this work non-commercially, and license their derivative works on different terms, provided the original work is properly cited, appropriate credit is given, any changes made indicated, and the use is non-commercial. See: http://creativecommons.org/licenses/by-nc/4.0/.

\section{REFERENCES}

1 Silver $\mathrm{H}$, Feldman P, Bilker W, et al. Working memory deficit as a core neuropsychological dysfunction in schizophrenia. Am J Psychiatry 2003;160:1809-16.

2 Barrantes-Vidal N, Aguilera M, Campanera S, et al. Working memory in siblings of schizophrenia patients. Schizophr Res 2007;95:70-5.

3 de Leeuw M, Kahn RS, Zandbelt BB, et al. Working memory and default mode network abnormalities in unaffected siblings of schizophrenia patients. Schizophr Res 2013;150:555-62.

4 Massuda R, Bücker J, Czepielewski LS, et al. Verbal memory impairment in healthy siblings of patients with schizophrenia. Schizophr Res 2013;150:580-2.
5 Liu Y, Wang G, Jin H, et al. Cognitive deficits in subjects at risk for psychosis, first-episode and chronic schizophrenia patients. Psychiatry Res 2019;274:235-42.

6 Lett TA, Voineskos AN, Kennedy JL, et al. Treating working memory deficits in schizophrenia: a review of the neurobiology. Biol Psychiatry 2014;75:361-70.

7 Malenka RC, Nestler EJ SE. Molecular neuropharmacology: a foundation for clinical neuroscience. 2 edn. New York: McGraw-Hill Medical, 2009: 313-21.

8 Harvey J, Finney S, Stewart L, et al. The relationship between cognition and sensation in determining when and where to void: the concept of cognitive voiding. BJU Int 2012;110:1756-61.

9 Baddeley A. The episodic buffer: a new component of working memory? Trends Cogn Sci 2000;4:417-23.

10 Hutter RRC, Allen RJ, Wood C. The formation of novel social category conjunctions in working memory: a possible role for the episodic buffer? Memory 2016;24:496-512.

11 Wang S, Allen RJ, Lee JR, et al. Evaluating the developmental trajectory of the episodic buffer component of working memory and its relation to word recognition in children. J Exp Child Psychol 2015;133:16-28.

12 Van Snellenberg JX, Girgis RR, Horga G, et al. Mechanisms of working memory impairment in schizophrenia. Biol Psychiatry 2016;80:617-26.

13 Boulet-Craig A, Robaey P, Lacourse K, et al. Visual short term memory related brain activity predicts mathematical abilities. Neuropsychology 2017;31:535-45.

14 Kopec CD, Erlich JC, Brunton BW, et al. Cortical and subcortical contributions to short-term memory for orienting movements. Neuron 2015;88:367-77.

15 Zhang L, Ran X, Li T, et al. Analysis of influencing factors of visual working memory in young adult patients with schizophrenia. Gen Psychiatr 2018;31:e100036.

16 Gan H, Song Z, Xu P, et al. A comparison study of working memory deficits between patients with methamphetamine-associated psychosis and patients with schizophrenia. Shanghai Arch Psychiatry 2018;30:168-77

17 Mayer JS, Bittner RA, Nikolić D, et al. Common neural substrates for visual working memory and attention. Neuroimage 2007;36:441-53.

18 Bor D, Duncan J, Wiseman RJ, et al. Encoding strategies dissociate prefrontal activity from working memory demand. Neuron 2003;37:361-7

19 Edin F, Klingberg T, Johansson P, et al. Mechanism for top-down control of working memory capacity. Proc Natl Acad Sci U S A 2009;106:6802-7.

20 Curtis CE, D'Esposito M. Persistent activity in the prefrontal cortex during working memory. Trends Cogn Sci 2003;7:415-23.

21 Sakai K, Rowe JB, Passingham RE. Active maintenance in prefrontal area 46 creates distractor-resistant memory. Nat Neurosci 2002;5:479-84

22 Nee DE, Jonides J. Neural correlates of access to short-term memory. Proc Natl Acad Sci U S A 2008;105:14228-33.

23 Bledowski C, Cohen Kadosh K, Wibral M, et al. Mental chronometry of working memory retrieval: a combined functional magnetic resonance imaging and event-related potentials approach. $J$ Neurosci 2006;26:821-9.

24 Bittner RA, Linden DEJ, Roebroeck A, et al. The when and where of working memory dysfunction in early-onset Schizophrenia-A functional magnetic resonance imaging study. Cereb Cortex 2015;25:2494-506.

25 Anticevic A, Repovs G, Barch DM. Working memory encoding and maintenance deficits in schizophrenia: neural evidence for activation and deactivation abnormalities. Schizophr Bull 2013;39:168-78.

26 Choi J-S, Park J-Y, Jung MH, et al. Phase-specific brain change of spatial working memory processing in genetic and ultra-high risk groups of schizophrenia. Schizophr Bull 2012;38:1189-99.

27 Schlösser RGM, Koch K, Wagner G, et al. Inefficient executive cognitive control in schizophrenia is preceded by altered functional activation during information encoding: an fMRI study. Neuropsychologia 2008;46:336-47.

28 Van Snellenberg JX, Torres IJ, Thornton AE. Functional neuroimaging of working memory in schizophrenia: task performance as a Moderating variable. Neuropsychology 2006;20:497-510.

29 Glahn DC, Ragland JD, Abramoff A, et al. Beyond hypofrontality: a quantitative meta-analysis of functional neuroimaging studies of working memory in schizophrenia. Hum Brain Mapp 2005;25:60-9.

$30 \mathrm{Kim} \mathrm{J}-\mathrm{J}$, Kwon JS, Park HJ, et al. Functional disconnection between the prefrontal and parietal cortices during working memory processing in Schizophrenia: $\mathrm{A}\left[{ }^{15} \mathrm{O}\right] \mathrm{H}_{2}$ O PET study. AJP 2003;160:919-23. 
31 Coleman MJ, Cook S, Matthysse S, et al. Spatial and object working memory impairments in schizophrenia patients: a Bayesian itemresponse theory analysis. J Abnorm Psychol 2002;111:425-35.

32 Park S, Püschel J, Sauter BH, et al. Visual object working memory function and clinical symptoms in schizophrenia. Schizophr Res 2003;59:261-8.

33 Silver H, Bilker WB. Similar verbal memory impairments in schizophrenia and healthy aging. Implications for understanding of neural mechanisms. Psychiatry Res 2015;226:277-83.

34 Gasbarri A, Pompili A, D'Onofrio A, et al. Working memory for emotional facial expressions: role of estrogen in humans and nonhuman primates. Rev Neurosci 2008;19:129-48.

35 Mendelson G. Diagnostic and statistical manual of mental disorders, fourth edition (DSM-IV). Aust N Z J Psychiatry 2009;29.

36 Kay SR, Fiszbein A, Opler LA. The positive and negative syndrome scale (PANSS) for schizophrenia. Schizophr Bull 1987;13:261-76.

37 Chan RCK, Shi Y-fang, Lai M-kin, et al. The temporal experience of Pleasure scale (TEPS): exploration and confirmation of factor structure in a healthy Chinese sample. PLoS One 2012;7:e35352.

38 Liu W-hua, Wang L-zhi, Zhu Y-hua, et al. Clinical utility of the SnaithHamilton-Pleasure scale in the Chinese settings. BMC Psychiatry 2012;12:184.

39 Tianmei S, Liang S, Yun'ai S, et al. The Chinese version of the personal and social performance scale (PSP): validity and reliability. Psychiatry Res 2011;185:275-9.

40 Gold JM, Queern C, lannone VN, et al. Repeatable battery for the assessment of neuropsychological status as a screening test in schizophrenia I: sensitivity, reliability, and validity. Am J Psychiatry 1999;156:1944-50.

41 Hobart MP, Goldberg R, Bartko JJ, et al. Repeatable battery for the assessment of neuropsychological status as a screening test in schizophrenia, II: convergent/discriminant validity and diagnostic group comparisons. Am J Psychiatry 1999;156:1951-7.

42 Kern RS, Nuechterlein KH, Green MF, et al. The MATRICS consensus cognitive battery, part 2: co-norming and standardization. Am J Psychiatry 2008;165:214-20.

43 Nuechterlein KH, Green MF, Kern RS, et al. The MATRICS consensus cognitive battery, part 1: test selection, reliability, and validity. Am J Psychiatry 2008;165:203-13.

44 Xu G, Huang YX, Yan W. Revision of the Chinese facial affective picture system, 2011.

45 Collins AGE, Albrecht MA, Waltz JA, et al. Interactions among working memory, reinforcement learning, and effort in value-based choice: a new paradigm and selective deficits in schizophrenia. Biol Psychiatry 2017;82:431-9.

46 Oishi Y, Yamakawa T, Nagasawa H, et al. Pure topographical disorientation in novel environments without anterograde amnesia: a case study. Neurocase 2019;25:177-86.

47 Sheehan MJ, Tibbetts EA. Specialized face learning is associated with individual recognition in paper wasps. Science 2011;334:1272-5.

48 Tsao DY, Freiwald WA, Tootell RBH, et al. A cortical region consisting entirely of face-selective cells. Science 2006;311:670-4.

49 Negrini M, Brkić D, Pizzamiglio S, et al. Neurophysiological correlates of Featural and spacing processing for face and Non-face stimuli. Front Psychol 2017;8:333.

50 Kume Y, Maekawa T, Urakawa T, et al. Neuromagnetic evidence that the right fusiform face area is essential for human face awareness: an intermittent binocular rivalry study. Neurosci Res 2016;109:54-62.

51 Huang W, Wu X, Hu L, et al. Revisiting the earliest electrophysiological correlate of familiar face recognition. Int $J$ Psychophysiol 2017;120:42-53.

52 Vakli P, Németh K, Zimmer M, et al. The electrophysiological correlates of integrated face and body-part perception. $Q$ J Exp Psychol 2017;70:142-53.

53 Lacey S, Stilla R, Deshpande G, et al. Engagement of the left extrastriate body area during body-part metaphor comprehension. Brain Lang 2017;166:1-18.

54 Downing PE, Jiang Y, Shuman M, et al. A cortical area selective for visual processing of the human body. Science 2001;293:2470-3.

55 Draht F, Zhang S, Rayan A, et al. Experience-dependency of reliance on local visual and Idiothetic cues for spatial representations created in the absence of distal information. Front Behav Neurosci 2017;11:92

56 Zhang X, Yao J, Lv Y, et al. An association study on the cognitive function and the cerebral grey matter volume of patients with FirstEpisode schizophrenia. Shanghai Arch Psychiatry 2018;30:154-67.

57 Sun C, Zhou P, Wang C, et al. Defects of gamma oscillations in auditory steady-state evoked potential of schizophrenia. Shanghai Arch Psychiatry 2018;30:27-38.

58 Zhang $\mathrm{H}$, Wang $\mathrm{Y}$, Hu Y, et al. Meta-Analysis of cognitive function in Chinese first-episode schizophrenia: MATRICS consensus cognitive battery (MCCB) profile of impairment. Gen Psychiatr 2019;32:e100043.

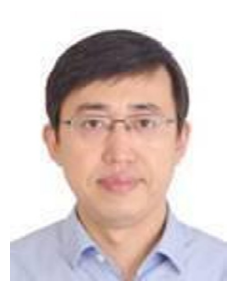

Yingjun Zheng obtained a bachelor's degree from Norman Bethune Health Science Center of Jilin University, China in 1998, and a MD degree from Niigata University, China in 2008. He has been working at the Affiliated Brain Hospital of Guangzhou Medical University, Guangzhou in China since 2011. Now he is working as a professor of Psychiatry and the clinical chief of Adult Psychiatry Ward at the Department of Psychiatry. In addition, he is also a committee member of the Psychiatric Association of Guangdong Province, the Chinese Association of rehabilitation medicine, and the Chinese society of rehabilitation medicine. His main research interests include bio-psychiatric research of schizophrenia, especially in research about neurophysiology, behaviour, brain imaging and biological associated biomarkers under visual and auditory tasks for the early detection and differential diagnosis of schizophrenia. 